\title{
Contrast sensitivity and acuity relationship in strabismic and anisometropic amblyopia
}

\author{
M ABRAHAMSSON AND J SJÖSTRAND \\ From the Department of Ophthalmology, University of Göteborg, Sahlgren's Hospital S-413 45 Göteborg, \\ Sweden
}

SUMMARY The contrast sensitivity function (CSF) and visual acuity were determined in children and adults with unilateral amblyopia due to strabismus or anisometropia with central fixation. The preschool children were examined repeatedly during occlusion treatment. All amblyopes had CSF deficits. The CSF was characterised by its peak value (the maximal sensitivity, $S_{\max }$, and the spatial frequency at which $S_{\text {max }}$ occurs, $F_{\text {max }}$ ) calculated by a single peak least-square regression method. The two amblyopic groups showed discrepancies in relationship of both $S_{\max }$ and $\mathrm{Fr}_{\max }$ versus visual acuity both initially and during treatment. The strabismic cases had a more marked visual acuity deficit in relation to the contrast sensitivity losses, whereas these parameters are affected similarly in anisometropic amblyopes. The relationship between recovery of visual acuity and CSF during the initial month of occlusion treatment was of prognostic significance for the outcome of visual acuity improvement.

Amblyopia is defined as an optically uncorrectable loss of vision, usually monocular, without demonstrable pathology in the posterior pole of the eye. This condition develops in early childhood and it affects up to $5 \%$ of the population. It is usually connected with strabismus or anisometropia. This visual degradation is treatable during childhood and the common treatment is occlusion therapy. The 'better' eye is occluded for longer or shorter periods.

Amblyopia has been studied by psychophysical tests in humans and in both psychophysical and electrophysiological tests in animals. Although there are great differences in the duration of critical or sensitive periods between animals and humans, animal models can provide a useful conceptual framework to follow. Furthermore, several behavioural aspects of amblyopia in the monkey are similar to human amblyopia in terms of aetiology, severity, and occurrence during a period early in life when the visual system is susceptible to abnormal input. ${ }^{1-3}$

In 1974 Ikeda and Wright suggested that the common aetiological basis for ambylopia caused by anisometropia and strabismus may be 'blur'. ${ }^{+}$In an anisometropic pair of eyes one of the eyes probably

Correspondence to $\mathbf{M}$ Abrahamsson, Department of Ophthalmology, Sahlgren's Hospital, S-413 45 Göteborg, Sweden. receives an input with deprived form and contour. There is no evidence that a strabismic eye receives a blurred image, whereas several studies indicate that abnormal binocular interaction is present in strabismus. The results from psychophysical studies in humans indicate that a common explanation for these two types of amblyopia ${ }^{5-\infty}$ is unlikely.

Studies of the effect of illumination ${ }^{8}$ on contrast sensitivity function (CSF) and apparent suprathreshold contrast ${ }^{9} 11$ in the two groups have indicated differences in the dysfunction in amblyopia due to strabismus and anisometropia. A threshold contrast comparison between these two groups also indicates differences. " " In general, the CSF from a patient with strabismic amblyopia is affected in the high frequency part, while the anisometropic patients have a CSF depressed over the whole frequency spectrum. These data support a theory whereby strabismic amblyopes have deficits mainly caused by spatial distortion in the central visual field while anisometropic amblyopia is caused by a generally impaired resolution..$^{13}$

In this study we have used a statistical method, Ushaped regression, ${ }^{14}$ to determine the maximum contrast sensitivity $\left(S_{\max }\right)$ and the spatial frequency at which it occurs $\left(\mathrm{Fr}_{\max }\right)$ in order to characterise the CSF. This method has proved to be a useful tool for 
evaluating CSF (Abrahamsson et al., in preparation). The aim of this study was to point out the differences in the threshold contrast sensitivity in relation to visual acuity between strabismic and anisometropic amblyopia before and during treatment by occlusion. We also investigated the possibility of predicting the outcome of amblyopia treatment from the initial (during the first month) changes in CSF and visual acuity.

\section{Material and methods}

Patients. Ten patients with strabismic amblyopia and 13 patients with anisometropic amblyopia together with 20 controls were examined. The controls were classified into two groups, 12 children (age 5-8 years) and eight adults (age 15-28 years). Anisometropia was defined as a refractive power difference of $1 \mathrm{D}$ or more in either pair of corresponding principal meridians in the two eyes and absence of any manifest deviation as judged by cover test in the primary position. From all patients or their parents informed consent was obtained.

Amblyopia was defined as a visual acuity quotient (the amblyopic eye acuity divided by the contralateral eye acuity) of $0 \cdot 8$ or less. Five of the strabismic amblyopes were children (age 5-8 years), who were examined before, after, and during treatment. The remaining five cases were adults (age 18-25 years). In the anisometropic amblyopia group were eight children (age 5-7 years) who were treated by occlusion and five adults (age 20-35 years).

The patients initially had a full ophthalmological examination, including cycloplegic refraction. All children with anisometropia and all children with strabismus except for one wore spectacles continuously for a period of at least one month before the initial testing. The children were treated thereafter by full-day occlusion of the dominant eye for up to five or six days a week during the initial period. The visual acuity (VA) and CSF were serially measured during the occlusion period, which continued until the maximum improvement was achieved. After the end of treatment the children were followed up by their ordinary ophthalmologist. The post-treatment visual acuity was tested at least one year after completed therapy. Visual acuity was assessed in the children by linear E optotypes (KIFA: MonoyerGranström) at $5 \mathrm{~m}$ with optimal correction. Ophthalmoscopy demonstrated that all the patients had clear media and no observable abnormalities in the posterior pole of the eye.

In all cases selected, fixation was considered central, though unsteady and slightly drifting in some of them.

Apparatus and procedure. Vertical sinusoidal grating was generated on a television display as previously described by Sjöstrand.'2 The space average luminance of the grating was $135 \mathrm{~cd} / \mathrm{m}^{2}$. The television was masked to subtend $1.7 \times 1.7$ degrees of visual angle, when viewed at a distance of $4 \mathrm{~m}$. Spatial frequency was varied between 1 and 32 cycles per degree. Pattern contrast was varied in $2 \mathrm{~dB}$ steps in the range 0.7 to 0.0007 . The contrast sensitivity (reciprocal to contrast) function was determined monocularly by the increasing contrast method.

Each test session started with a visual acuity testing. Then the child had a break as he walked to an other examining room for the CSF test. The CSF was tested by a game where the child chose among glass balls and wooden dolls of different sizes to match the spatial frequency of the grating when it just becomes detectable. Breaks were allowed whenever the child lost interest in the test. All tests were performed by the authors. The children were tested before and repeatedly after the start of the patching.

Statistics. The co-ordinates of the peak of the CSF (maximum sensitivity, $S_{\max }$, and the frequency at which it occurs, $\mathrm{Fr}_{\max }$ ) were calculated by a single peak least square fit estimation. ${ }^{14}$ The statistical calculation was based on the assumption that the CSF monotonously rises to a maximum and then monotonously (without local maxima or minima) declines. The CSF cut-off frequency was calculated by extrapolating a linear regression of the high-frequency limb of the CSF to contrast sensitivity level 1.0 .

Diagnostic indices. In this explorative study several simple, potentially valuable indices can be calculated (Table 1). To compare these indices with respect to their discriminating ability two limits were determined so that $10 \%$ (two out of 20 ) of the observed normal eyes would exceed these limits. Thus the specificity (the proportion of correctly classified normals) will be approximately $90 \%$. The sensitivity (the proportion of correctly classified patients) in this sample is determined to be the proportion of the observed cases exceeding the limits. The sensitivity values for different indices are reported in Table 1 . As could be expected, VA separates amblyopes from controls very well, since acuity is used as the definition of amblyopia.

Other types of diagnostic indices are those where the discrimination is between the different groups of patients. To see how well some diagnostic indices could separate the two amblyopic groups from each other a limit is set for each index so that patients on one side of the limit are classified as strabismic and on the other as anisometropic. The limit for each index is determined in such a way that the percentage of correctly classified strabismic patients equals the percentage correctly classified anisometropic patients, and this common percentage is reported in 
Table 2. Tables 1 and 2 together demonstrate two different aspects of a diagnostic index that are equally important.

\section{Results}

The distribution of the sensitivity maximum versus acuity of the amblyopic eyes of 23 patients together with 20 normal persons is presented in Fig. 1. The data from the initial test session were used. The data points representing the amblyopic eyes were gathered into two partly separated clusters. The strabismic amblyopes show an $\mathrm{S}_{\max }$ value rather independent of visual acuity, while in the anisometropic amblyopia there is a clear relationship between visual acuity and $S_{\max }$. In general the $S_{\max }$ value for the strabismic group is higher than for the anisometropic amblyopia group.

Since the anisometropic group initially had lower visual acuity levels than the strabismic group, we compared $S_{\max }$ for the two groups at three selected visual acuity levels $(0 \cdot 2,0 \cdot 3$, and $0 \cdot 5)$. Seven strabismic patients and 10 anisometropic patients had a visual acuity at at least one of the selected acuity levels at the initial session or at some occasion during treatment. In Fig. 2A the results are presented and the data from each patient are used only once. In comparison the anisometropic group had lower values of $S_{\max }$ than the strabismic patients. The distribution of the $\mathrm{Fr}_{\max }$ (Fig. 2B) at the same acuity levels showed a tendency for a more pronounced shift of the peak towards lower frequencies in the anisometropic group compared with the strabismic group.

The $S_{\max }$ calculated from the CSFs obtained during treatment of the children are presented in Fig. 3A.

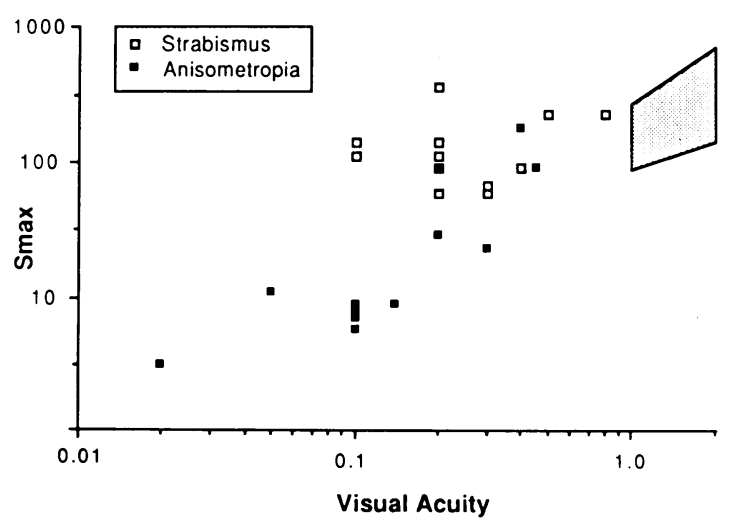

Fig. 1 The relationship between visual acuity and $S_{\max }$ in strabismic and anisometropic amblyopia. The two amblyopic groups are indicated by filled (anisometropia) and open squares (strabismus). The dotted area represents the normal range.

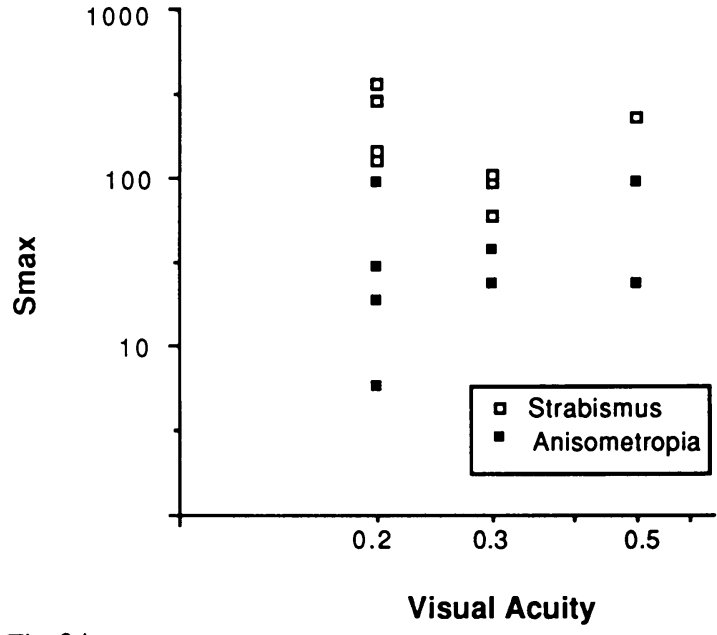

Fig. 2A

The similarity to the data in Figs. 1 and 2 is notable. The anisometropic patients have an increase in both acuity and $\mathrm{S}_{\max }$, while the strabismic patients show only an increasing visual acuity. In strabismic cases the $S_{\max }$ are at a near normal level independent of the acuity. The $\mathrm{Fr}_{\max }$ from the same sessions are presented in Fig. 3B. The strabismic group shows less variability in the $\mathrm{Fr}_{\max }$ during treatment than the anisometropic group. Grating acuity (cut-off frequency in this case) is usually compared with visual

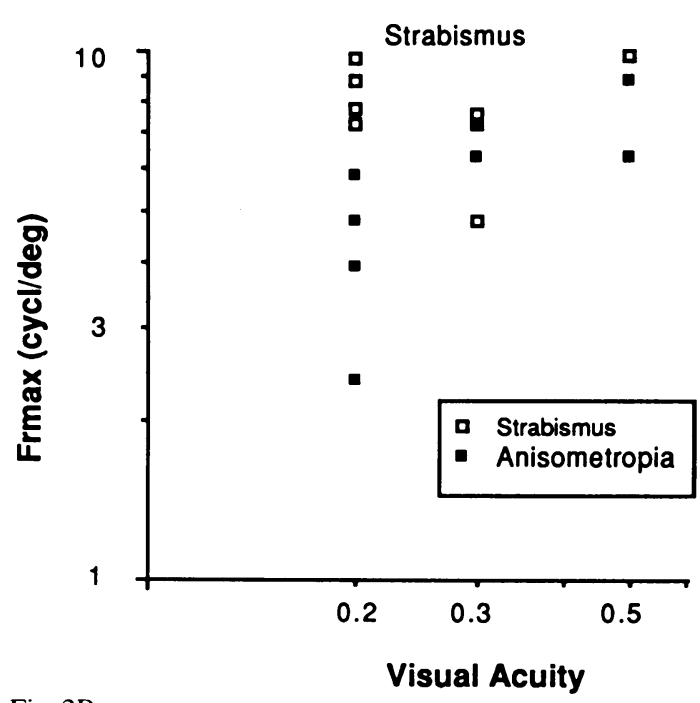

Fig. 2B

Fig. 2 A: The $S_{\max }$ of the two amblyopic groups at three selected visual acuity levels is presented $\mathrm{B}$ : The Fr $r_{\text {max }}$ at the same three visual acuity levels are presented. The same symbols as in Fig. 1 are used. 


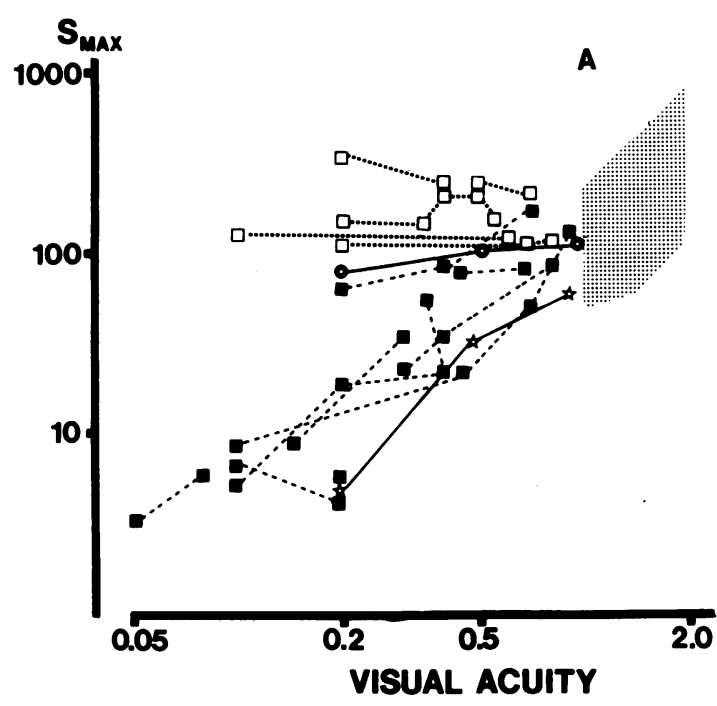

Fig. 3A

acuity by the equation $\mathrm{VA}=(\mathrm{GA}) / 30$. The results in our study show that cut-off frequencies in general are higher than the visual acuity would predict (results not shown).

$\mathbf{B}$

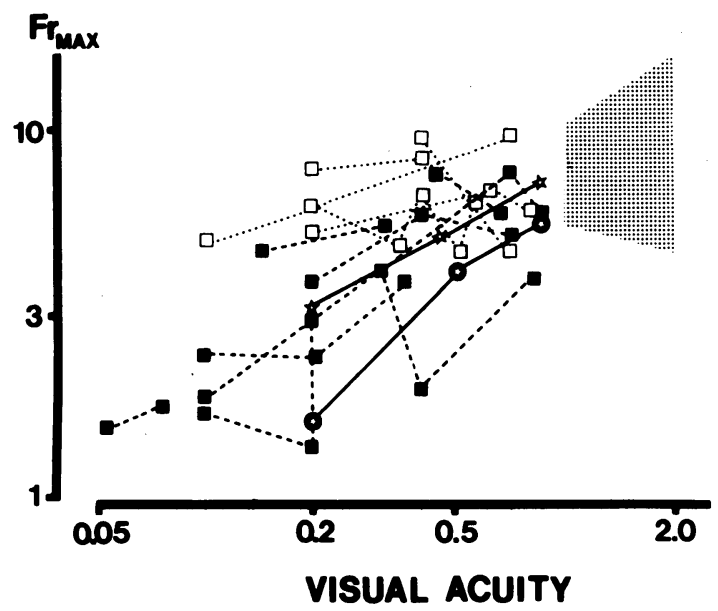

Fig. 3B

Fig. 3 A: $S_{\max }$ versus visual acuity during amblyopia treatment. The data from each patient are connected by $a$ dotted (strabismus) or dashed (anisometropia) line. In all cases the initial value is the one closest to the lower left corner. For the amblyopes the same symbols as in Fig. 1 are used. Stars in circles connected by a solid line indicate a typical retinal detachment patient during recovery, and stars connected by a solid line indicates data from a typical case of optic neuropathy during recovery. The normal range is indicated by the dotted area B: The same symbols are used where the $F R_{\max }$ versus visual acuity is presented.
Table 1 The ability of dignostic indices to separate amblyopic patients from normals measured as a percentage of correctly classified amblyopes and normals

\begin{tabular}{lr}
\hline Visual acuity & 100 \\
$\mathrm{~S}_{\max }$ & 56 \\
$\mathrm{~S}_{\text {max }} / \sqrt{\mathrm{VA}}$ & 84 \\
Cut-off frequency & 72 \\
Cur-off frequency $/ \sqrt{\mathrm{VA}}$ & 96
\end{tabular}

Table 2 The ability of diagnostic indices correctly to classify strabismic and anisometropic amblyopia: the percentage correctly classified amblyopes

\begin{tabular}{ll}
\hline Visual acuity & 62 \\
$\mathrm{~S}_{\max }$ & 62 \\
$\mathrm{~S}_{\max } / \sqrt{\mathrm{VA}}$ & 76 \\
Cut-off frequency & 58 \\
Cut-off frequency $/ \sqrt{\mathrm{VA}}$ & 58 \\
\hline
\end{tabular}

Visual acuity, $S_{\max }$, and cut-off frequency were used to calculate diagnostic indices. In Table 1 the ability of different diagnostic indices to separate normals from amblyopes is presented. Since visual acuity loss is used in the definition of amblyopia and a VA of 1.0 or better is a part of the definition of normals we found, as can be expected, that VAs classify all amblyopes and normals $(100 \%)$ correctly. The two CSF parameters ( $S_{\max }$, cut-off frequency) had lower diagnostic ability to differentiate amblyopes from controls. Table 2 indicates the ability of diagnostic indices to separate the two groups of amblyopes from each other. We found that $\mathrm{S}_{\max } / \sqrt{\mathrm{VA}}$ was the index that best separated the two groups of amblyopes from each other $(76 \%$ of the amblyopes were correctly classified as strabismic or anisometropic amblyopia). The same index has been used to separate patients with retinal detachment from those with optic neuropathy (Abrahamsson et al., in preparation).

The changes in visual acuity and CSF (represented by $S_{\max }$ and cut-off frequency) in the initial phase (first month) of the occlusion therapy have been studied. A high end-of-treatment visual acuity was the criterion we used to define a successful treatment. In four cases there was an acuity loss between end of treatment and the time at which the posttreatment acuity was obtained.

In Fig. 4 we have expressed the initial acuity changes as the percentage of increase of letter and grating acuity between the pretreatment values and the acuities obtained after one month of treatment. With this construction the number 0 indicates that the value obtained after one month is equal to the initial value and 100 means a doubling of the initial value. The data indicate that prognosis for a successful occlusion therapy improves as the VA percentage 


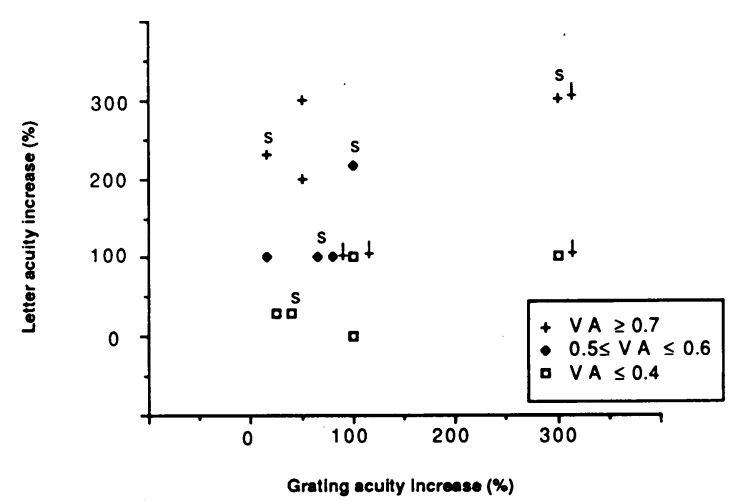

Fig. 4 The changes in $V A$ and $G A$ during the first month of occlusion therapy. The increase in letter and grating acuity during the first month is calculated as a percentage where 100 indicates that the value measured after one month of treatment is twice the initial value. The data are classified into three groups according to the visual acuity at the end of treatment. The four patients with a post-treatment letter acuity lower than the end of treatment acuity are marked with an arrow, and the strabismic cases are marked with an $\mathrm{S}$.

becomes comparably larger than the GA percentage, since all patients with a visual acuity $\geqslant 0.5$ at the end of treatment fulfilled the criteria VA versus GA increasing ratio $\geqslant 1 \cdot 0$.

In all four cases with an acuity loss during the period following the end of treatment and the final evaluation of post-treatment letter acuity the letter versus grating acuity increasing ratio was $\leqslant 1 \cdot 1$.

Owing to the small sample size and the differences in mean pretreatment visual acuity between the groups it was not possible in our small sample to find any relationship between the letter and grating acuity increase and age or type of amblyopia.

\section{Discussion}

Several studies have shown that there are discrepancies in CSF obtained in strabismic or anisometropic $^{51112}$ amblyopes. Strabismic amblyopia is generally characterised by a high-frequency decrease in the CSF, while the CSFs of anisometropic patients are affected at all spatial frequencies. A difference in the apparent suprathreshold contrast between the two groups has also been reported. " 1 In this study we have pointed out specific CSF discrepancies between the two groups. In strabismic patients the maximum point of the CSF is relatively uncorrelated with the visual acuity level. Even when the visual acuity is markedly reduced, the CSF curve is essentially normal from low frequencies to the maximum point, while the steepness of the high-frequency limb varies. The anisometropic amblyope generally has a CSF that is subnormal at all spatial frequencies. The maximum point is markedly shifted towards lower frequencies, and as acuity decreases the sensitivity declines simultaneously. There is no obvious relationship between $S_{\max }$ from the initial testing versus visual acuity from the same session in strabismic amblyopes whereas a clear relation is demonstrable in the anisometropic amblyopes (Fig. 1 ). When we study individual cases during treatment, this phenomenon, discrepancy in the $\mathrm{S}_{\max } / \sqrt{\mathrm{VA}}$ relation, is clearly shown (Fig. 3).

The use of the data from the initial test session can introduce a systematic error, since the untreated anisometropic amblyopes in general had a lower visual acuity than the untreated strabismic amblyopes. In order to get a fair estimation of the differences in the VA versus $S_{\text {max }}$ relation between the two groups we selected three visual acuity levels (Fig. 2). This analysis showed no significant differences between the results in Figs. 1 and 2 and confirmed the discrepancy between the two groups of amblyopes. The $S_{\max }$ of the anisometropic patients at a given acuity level is thus generally lower than that of the strabismic patients. There is a preferential loss of VA in strabismic amblyopia that resembles the vernier discrimination data presented by several authors. ${ }^{6131516}$ These reports suggested that spatial distortion is the primary cause of the loss of visual acuity in strabismic amblyopia. In anisometropic amblyopia spatial distortion can be demonstrated, but impaired resolution seems to be the major factor.

In a study (in preparation) we found that some of the parameters of central vision used in this study $\left(\mathrm{S}_{\max }, \mathrm{Fr}_{\max }\right.$, and VA) are useful in separating two groups of patients with retinal detachment and optic neuropathy respectively. Similarly, these parameters can be used as diagnostic indices for differentiating between two subgroups of amblyopia in the present study (Tables 1,2$)$. The relationship between $S_{\max }$ and VA in the strabismic patients data are similar to those of the retinal detachment patients, and the data of the anisometropic patients correspond to those of the patients with optic neuropathy. These findings concur with those of other studies ${ }^{5-x}$ showing that there are differencies in the neural basis of the two forms of amblyopia. Our present results, however, do not contradict the theory proposed by Flom and Bedell ${ }^{17}$ and supported by Hess and Pointer ${ }^{18}$ that the strabismic patients have abnormalities mainly in the central visual field, and the anisometropic amblyopia affects the whole visual field.

The relationship between the changes in VA and cut-off frequency during the first month of treatment and final visual acuity obtained at the end of the occlusion therapy have been used to study the possibility of establishing criteria for prognosis in 
relation to treatment. We found that a high VA at the end of treatment was correlated with a rapid and marked increase of the letter acuity during the first month of treatment. The data presented in Fig. 4 for the first month of treatment also indicate that a relative VA increase greater than the relative GA increase is correlated with a favourable prognosis. In all amblyopic patients with a post-treatment (obtained at least one year after end of treatment) VA of 0.5 or better the relative VA increase is greater than the relative GA increase. All patients with posttreatment $\mathrm{VA}<0.5$ and all patients with acuity loss after treatment ended were in the group with a relative VA increase less or approximately equal to the relative $\mathrm{GA}$ increase.

Our data indicate that the CSF versus acuity relationship gives some prediction of the outcome of occlusion therapy in individual amblyopic patients. One of the important factors seems to be the acuity change during the first month of treatment. The relation cut-off frequency change versus VA change ought to be evaluated only in those cases where there is no or a limited increase in VA during the first month. A small GA relative to the visual acuity increase during the first month seems to correlate with better end-of-treatment VA than does a large GA increase in combination with the same acuity increase. The same conclusion has been presented by Dale, ${ }^{19}$ who studied the relationship between acuity obtained by single letters and linear acuity.

In summary, there are differences in the CSF and even more pronounced differences in the relation VA versus CSF between anisometropic and strabismic amblyopia. These differencies can also be demonstrated in individual cases during treatment The findings support the theories which claim that there are differences in the neural basis of functional impairment in these two conditions. We have also found that the changes of CSF and acuity during the first month of treatment are predictive of the outcome of the occlusion therapy.

This study was supported by the Swedish Medical Research Council (grant 02226), Handlanden $H$ Svensson Foundation, and the Carmen and Bertil Régner Research Foundation. We will also thank Marianne Frisén for statistical assistance and Dr K Norrsell for valuable advice and constructive criticism.

\section{References}

1 Blakemore C. Vital-Durand F. Development of the neural basis of visual acuity in monkeys. Speculation on the origin of deprivation amblyopia. Trans Ophthalmol Soc UK 1979; 99: 363-8.

2 von Noorden GK, Dowling JE. Experimental amblyopia in monkeys II. Behavioral studics in strabismic amblyopia. Arch Ophthalmol 1970; 84: 215-20.

3 Smith III EL, Harwerth RS, Crawford MLJ. Spatial contrast sensitivity deficits in monkeys produced by optically induced anisometropia. Invest Ophthalmol Vis Sci 1985; 26: 330-42.

4 Ikeda $\mathrm{H}$, Wright MJ. Is amblyopia duc to inappropriate stimulation of the sustained pathway during development. Brit $J$ Ophthalmol 1974; 58: 165-75.

5 Hess RF, Howell ER. The threshold contrast sensitivity function in strabismic amblyopia: Evidence for a two type classification. Vision Res 1977; 17: 1049-55.

6 Levi DM, Klein S. Differences in vernier discrimination for gratings between strabismic and anisometropic amblyopes. Invest Ophthalmol Vis Sci 1982; 23: 398-407.

7 Hess RF, Bradley A, Piotrowski L. Contrast-coding in amblyopia I. Differences in neural basis of human amblyopia. Proc $R$ Soc Lond (Biol) 1983; 217: 309-30.

8 Hess RF, Campbell FW, Zimmern R. Differences in the neural basis of human amblyopias: The effect of mean luminance. Vision Res 1980; 20: 295-305.

9 Hess RF, Bradley A. Contrast perception above threshold is only minimally impaired in human amblyopia. Nature 1980; 287: 463-4.

10 Levi DM, Harwerth RS, Manny RE. Suprathreshold spatial frequency detection and binocular interaction in strabismic and anisometropic amblyopia. Invest Ophthalmol Vis Sci 1979; 18: 714-25.

11 Campos EC, Prampolini ML, Gulli R. Contrast sensitivity differences between strabismic and anisometropic amblyopia: objective correlate by means of visual evoked responses. Doc Ophthalmol 1984; 58: 45-50.

12 Sjöstrand J. Contrast sensitivity in children with strabismic and anisometropic amblyopia. A study of effect of treatment. Acta Ophthalmol (Kbh) 1981; 59: 25-34.

13 Bedell HE, Flom MC. Monocular spatial disorder in strabismic amblyopia. Invest Ophthalmol Vis Sci 1981; 20: 263-8.

14 Frisén M. U-shaped regression. COMPSTAT Proc comput statistics 1980; 4: 304-7.

15 Bedell HE, Flom MC, Barbeito R. Spatial aberration and acuity in strabismus and amblyopia. Invest Ophthalmol Vis Sci 1985; 26: 909-16.

16 Stuart JA, Burian HM. A study of separation difficulty. Am J Ophthalmol 1962; 53: 471-7.

17 Flom MC, Bedell HE. Identifying amblyopia using associated conditions: acuity and nonacuity features. Am J Opt Physiol Opt 1985; 62: 153-60.

18 Hess RF, Pointer JS. Differences in the neural basis of human amblyopia. The distribution of the anomaly across the visual field. Vision Res 1985; 25: 1577-94.

19 Dale RT. Fundamentals of ocular mobility and strabismus. New York: Grune and Stratton, 1982: 132-60.

Accepted for publication 27th November 1986. 